\title{
Relevance of assessing quadriceps endurance in patients with COPD
}

\author{
C. Coronell*, M. Orozco-Levi*, R. Méndez*, A. Ramírez-Sarmiento*, J.B. Gáldiz\#, J. Gea*
}

Relevance of assessing quadriceps endurance in patients with COPD. C. Coronell, M. Orozco-Levi, R. Méndez, A. Ramírez-Sarmiento, J.B. Gáldiz, J. Gea. (C)ERS Journals Ltd 2004.

ABSTRACT: The aims of this study were to investigate whether the impairment in endurance of limb muscles is a general finding in chronic obstructive pulmonary disease (COPD) patients, affecting even those with mild-to-moderate disease or relatively normal physical activity. In addition, this study aimed to determine the physiopathology of exhaustion in local endurance tests and whether the reduction in quadriceps endurance can be predicted from muscle strength measurements.

A total of 75 volunteers were assigned to one of two groups according to pulmonary function tests: COPD patients or healthy age-matched controls. Functional assessment included both quadriceps strength (maximum voluntary contraction (QMVC)), and quadriceps endurance (contractions against a load equivalent to $10 \%$ QMVC until task failure or for up to a limiting time of $30 \mathrm{~min}$ (QTlim)).

COPD patients showed a decrease of $\sim 43 \%$ in QMVC and $\sim 77 \%$ in QTlim compared with controls. Task failure occurred only in COPD patients and was due to muscle fatigue, since limiting symptoms were associated with a decrease in the median frequency of quadriceps electromyographical signal and a reversible decrease in QMVC. The impairment in skeletal muscle endurance was present even in patients with mild-tomoderate airflow obstruction and individuals with relatively normal physical activity, and was irrespective of lung function variables, anthropometrical data or quadriceps strength.

Peripheral muscle endurance was impaired in chronic obstructive pulmonary disease patients, even in those with relatively normal physical activity and mild-to-moderate airflow obstruction. This impairment associated with an early onset of muscle fatigue and could not be predicted from the severity of the disease or the reduction in quadriceps strength.

Eur Respir J 2004; 24: 129-136.
* Muscle and Respiratory Research Unit, Municipal Institute of Medical Research, Servei de Pneumologia, Hospital del Mar, CEXS-Universitat Pompeu Fabra, Barcelona, Catalonia, and "Unit of Respiratory Pathology, Cruces Hospital, Baracaldo, Basque Country, Spain.

Correspondence: M. Orozco-Levi, Servei de Pneumologia, Hospital del Mar, Passeig Maritim 25-27, Barcelona 08003, Spain.

Fax: 34932213237

E-mail: morozco@imim.es

Keywords: Chronic obstructive pulmonary disease

endurance

lower limb muscles

strength

weakness

Received: July 92003

Accepted after revision: February 252004

This study was supported by Red Respira (RTIC C03/11, Fondo de Investigación Sanitaria, Instituto de Salud Carlos III), Sociedad Española de Neumología Cirugía Torácia, Ministerio de Educación, Cultura y Deporte de España, Grant Code 72.129.052.
The capacity to perform physical exercise is determined by multiple organs and systems [1,2]. Patients with chronic obstructive pulmonary disease (COPD) undergo several degrees of impairment in the capacity to perform physical activities (e.g. exercise and daily physical activity (PA)) [3-6]. Impairment of their ventilatory function represents the main limiting factor for exercise capacity in most patients. However, other extrapulmonary factors also play a significant role in limiting exercise. MALTAIs et al. [7] showed that peripheral muscle dysfunction (i.e. early onset of lactic acidosis) appears to be involved in early exercise termination in severe COPD patients during an incremental exercise test. This skeletal muscle dysfunction shows clinical relevance because it is related to an increased need for medical assistance and decreased survival of COPD patients [8-11]. In addition, a limited improvement in exercise capacity has been demonstrated when COPD patients receive a pulmonary or combined heart-lung transplant [12-14].

Skeletal muscles have two functional characteristics: strength and endurance. Reduced maximal muscle strength was demonstrated in COPD patients, which was found to be a significant contributor to work capacity limitation $[3,15]$. At present, few data are available on skeletal muscle endurance

For editorial comments see page 6.
[16] and, for the assessment of peripheral muscle performance, isolated muscle strength alone is too restrictive. In a group of 17 COPD patients, SERRES et al. [16] showed positive correlations between specific (local) quadriceps endurance and the PA score, degree of airflow obstruction (i.e. forced expiratory volume in one second (FEV1)) and arterial oxygen tension $\left(\mathrm{Pa}, \mathrm{O}_{2}\right)$. Their findings clearly illustrated that impaired skeletal muscle endurance in COPD patients relates to altered lung function and associated physical inactivity. It was impossible for the authors to determine the respective components of bronchial obstruction and deconditioning in altering skeletal muscle performance. Therefore, studies comparing healthy control subjects and COPD patients according to PA are needed to investigate the role of bronchial obstruction in altering skeletal muscle function. Moreover, to the authors' knowledge, no reports exist describing the electromyographical changes or the cardiorespiratory responses while performing a local quadriceps exercise. The aim of the present study was to further characterise quadriceps muscle endurance in stable COPD patients determining: 1) whether the impairment in endurance of limb muscles is a general finding in COPD patients affecting those with mild-to-moderate disease, normal weight and relatively preserved PA; 2) the physiopathology of exhaustion in a local quadriceps endurance test; and 3) whether the reduction in quadriceps endurance can be 
predicted from strength measurements of the muscle. A local, single limb, submaximal exercise was performed in the presence of a normalised load (equivalent to $10 \%$ of quadriceps maximal strength). Symptoms and physiological responses during the exercise were also analysed.

\section{Methods}

\section{Study population}

A total of 75 male volunteers were assigned to one of two study groups, according to both age and pulmonary function tests. The first group $(n=36)$ included patients with COPD (aged $67 \pm 8$ yrs; FEV1 $18-76 \%$ pred) lacking comorbidities. The second group $(n=39)$ included age-matched healthy individuals (aged $61 \pm 10$ yrs; FEV1 $84-119 \%$ pred). Diagnosis of COPD was performed by assessment of the signs and symptoms of chronic bronchitis and/or pulmonary emphysema, time-exposure to cigarette smoke, and functional criteria of chronic and irreversible airflow obstruction (FEV1/forced vital capacity $<70 \%$, FEV $1<80 \%$ pred, and $<12 \%$ change in FEV1 following $400 \mu \mathrm{g}$ of inhaled salbutamol) [17, 18]. All patients were clinically stable for $\geqslant 4$ months from the last exacerbation and received inhaled bronchodilators. The presence of cardiopathies, metabolic diseases or joint dysfunctions were considered as exclusion criteria. The control group included healthy volunteers prospectively recruited from the general population. Healthy status was defined as the absence of known diseases as assessed by a systematic medical interview, general physical examination (performed by experienced physicians from the authors' group), and normal pulmonary function. The Research Committee of Human Investigation at the Municipal Institute for Medical Research, Hospital del Mar (Barcelona, Spain) approved the study and informed written consent was obtained from each participant after a full explanation of the purposes and characteristics of the study.

\section{Study design}

This was a cross-sectional study. The selected volunteers were examined and subsequently underwent tests measuring nutritional status, pulmonary function, inspiratory muscle strength, hand grip strength, and daily PA. Functional assessment of the muscle included both maximum quadriceps strength measured during maximum voluntary contractions (QMVC), and quadriceps endurance time (QTlim) defined as the elapsed time required performing contractions against a load equivalent to $10 \%$ QMVC until task failure, as described below.

\section{Measurements}

Anthropometry and nutritional evaluation. Levels of serum albumin, cholesterol and transferrin were determined as biological parameters. Body weight, body mass index (BMI), and length and perimeter of the thigh of both legs were evaluated as anthropometric parameters.

Daily physical activity. Daily PA was assessed by the Baecke's questionnaire [19] modified for elderly people [20, 21]. This questionnaire evaluated household activities, sports activities and other physically active leisure-time activities during the previous year, and gave an overall PA score. The subjects were asked to describe the type of activity, hours per week participation and the period of the year in which the activity was normally performed. All activities were classified according to posture and movement. An intensity code based on net energetic costs of activities according to BINK et al. [22] was used to classify each activity. The method evaluates the activity of older subjects as high, medium or low. With this method, subjects who obtained a score $\leqslant 9$ were classified as having low PA, and thus "sedentary" [16, 20, 21].

Pulmonary and respiratory muscle function. Pulmonary and respiratory muscle function was assessed by forced spirometry [23] (Datospir 900; SIBEL, Barcelona, Spain), static lung volumes, airways resistance and carbon monoxide transfer factor [24] (Masterlab, Jaeger, Würzburg, Germany). Reference values used were those for the Mediterranean population [25]. Arterial blood samples were obtained from the radial artery of the nondominant arm for partial pressure of oxygen and partial pressure of carbon dioxide measurements using conventional polarographic techniques (Rapidlab 860; Chiron/diagnostic, Wuppertal-Barmen, Germany). Maximum inspiratory pressures at the mouth were included as measures of inspiratory and expiratory muscle strength, respectively. Reference values used were those obtained by MORALES et al. [26] for a Mediterranean population.

Handgrip strength. Handgrip strength was assessed using a handheld dynamometer (Biopac Systems, Schooner, CA, USA) connected to a digital polygraph. The maximum voluntary contraction of the flexor muscles of both dominant and nondominant hands was assessed, and the highest value of three reproducible ( $<5 \%$ variability between values) manoeuvres was used in the analysis [27, 28].

Quadriceps muscle function. Function of the quadriceps muscle was evaluated by muscle strength and endurance of the dominant leg during specific (local) single leg exercises at an exercise bench. Specifically, the strength of the muscle was assessed through isometric maximum voluntary contraction (QMVC) of the dominant lower limb while the patients were seated with both trunk and thigh fixed on a rigid support of an exercise platform (Domyos HGH 050; Decathlon, Lille, France). The highest value from three brief (3 s) reproducible QMVC manoeuvres ( $<5 \%$ variability between values) was included in the analysis. The reference values included were those from DECRAMER et al. [29]. Endurance of the quadriceps was assessed following the method described by SERRES et al. [16]. The load was modified to $10 \%$ of the QMVC, normalised the extension of the thigh and recorded electromyography (EMG) activity. The primary outcome variable was QTlim. The volunteers performed intermittent knee extensions on the exercise platform. Strength was quantified by an isometric dynamometer (Biopac Systems) connected to a digital polygraph (Biopac Systems). The axis of rotation, the range of displacement and the point of distal attachment were identical for all individuals. The contraction pattern was normalised at a frequency of 12 extensions per min, against an external load equivalent to $10 \%$ QMVC, allowing $2 \mathrm{~s}$ for contraction and $3 \mathrm{~s}$ for relaxation. The contraction cycling was imposed by using a digital audio signal (Joggler Plus 4.8.1; Leepoware, San José, CA, USA). The tension-time (TT) index (TTQ) for the quadriceps was calculated from the following formula:

$$
\mathrm{TT} \mathrm{Q}=(T \mathrm{C} / T \text { TOT }) *(\mathrm{~W} / \mathrm{QMvC})
$$

where $T \mathrm{C}$ is the contraction time, $T$ TOT is the duration of each cycle and $\mathrm{W}$ is the external load (weight). The perception of symptoms on the exercised leg and dyspnoea were assessed as secondary outcome variables. Two visual analogical scales were used at 2-min intervals until task failure, which was defined by one of two criteria [30]; first, the inability to 
maintain leg extension equivalent to $\geqslant 80 \%$ of the maximum extension during three consecutive contractions, and secondly, the inability to continue the exercise due to invalidating symptoms. The exercise was finished if the volunteers reached a QTlim of $30 \mathrm{~min}$ without succumbing to task failure. The analysis of the QTlim test also included two variables related to the potential appearance of muscle fatigue. The first variable analysed was a change in the EMG activity and was assessed by calculating the EMG median frequency $(f \mathrm{EMG})$ within each cycle of contraction-relaxation of the quadriceps [31]. For this purpose, a continuous electromyographic recording was obtained by using bipolar surface electrodes in accordance with conventional techniques [32]. Electrodes were placed over the vastus lateralis muscle at approximately the midpoint between the head of the greater trochanter and lateral condyle of the femur. EMG signal was amplified in a frequency band ranging 100-2,000 Hz. Median frequency was determined by performing a fast fourier transformation of the three EMG recordings of the contraction at the beginning of each quintile of the whole QTlim record followed by integration and then normalisation of the spectrum. The second variable assessed was the potential change in the QMVC measured after the task failure. Ventilatory response, oxygen consumption $\left(V^{\prime} \mathrm{O}_{2}\right)$ and production of carbon dioxide $\left(V^{\prime} \mathrm{CO}_{2}\right)$ were also continuously measured during the endurance test (Oxycon Alpha; Jaeger). Heart rate and pulse oxymetry were recorded using a pulseoxymeter (MM205; Medical Artema Bd, Sundbyberg, Sweden).

\section{Statistical analysis}

All parameters were checked for normality of distribution. Differences between COPD patients and the healthy control group were assessed using an unpaired t-test for independent samples. Changes in $f$ EMG were assessed using the analysis of variance test for repeated measures. Association between continuous variables was evaluated using the Pearson's coefficient and linear regression analysis.

\section{Results}

\section{Nutritional status and anthropometrical data}

All nutritional variables were within the reference values in both study groups (table 1). Body weight, BMI and thigh length were similar within the study groups. Thigh perimeter tended to be smaller in COPD patients (mean difference $\sim 4 \mathrm{~cm}$ ) when compared with aged-matched controls. However, this difference was far from statistical significance.

\section{Pulmonary function}

All control individuals showed normal pulmonary function tests. The group of patients with COPD included a wide range of airflow obstruction (FEV1 18-76\% pred). A total of 35 patients $(97 \%)$ showed pulmonary air trapping (defined as residual volume:total lung capacity $>35 \%)$, 18 patients $(50 \%)$ showed pulmonary hyperinflation (defined as total lung capacity $>120 \%$ pred), and 23 patients $(72 \%)$ showed a decreased $\mathrm{CO}$ transfer factor (table 1). A total of 26 patients showed hypoxaemia $\left(\mathrm{Pa}, \mathrm{O}_{2}<10.6 \mathrm{kPa}\right)$, whereas eight fulfilled criteria of chronic respiratory failure $\left(\mathrm{Pa}, \mathrm{O}_{2}<8.0 \mathrm{kPa}\right)$.

\section{Daily physical activity scores}

Daily PA scores ranged 2.6-26.6 with a median value of 11 . Thirteen patients scored $<9$ in the PA questionnaire. No
Table 1.-General characteristics of the study population

Healthy elderly COPD patients controls

\begin{tabular}{lcc}
\hline Subjects n & 39 & 36 \\
Age yrs & $65 \pm 9$ & $67 \pm 8$ \\
Weight kg & $71 \pm 8$ & $69 \pm 16$ \\
Height cm & $166 \pm 5$ & $165 \pm 5$ \\
BMI kg·m ${ }^{-2}$ & $25.7 \pm 2.6$ & $25.2 \pm 5.8$ \\
FEV $\%$ pred & $92 \pm 7$ & $36 \pm 14^{* *}$ \\
FEV $1 / \mathrm{FVC} \%$ & $72 \pm 4$ & $46 \pm 10^{* *}$ \\
$\mathrm{TLC} \%$ pred & $88 \pm 15$ & $113 \pm 30$ \\
$D \mathrm{~L}, \mathrm{CO} \%$ pred & $90 \pm 18$ & $64 \pm 25^{* *}$ \\
$P \mathrm{a}, \mathrm{O}_{2} \mathrm{mmHg}$ & $\mathrm{ND}$ & $64 \pm 11$ \\
$P a, \mathrm{CO}_{2} \mathrm{mmHg}$ & $\mathrm{ND}$ & $47 \pm 8$ \\
$P \mathrm{I}, \mathrm{max} \mathrm{cmH} \mathrm{H}_{2} \mathrm{O}$ & $-110 \pm 40$ & $-39 \pm 24^{* *}$ \\
$P \mathrm{I}, \mathrm{max} \%$ pred & $117 \pm 35$ & $38 \pm 21^{* *}$ \\
$\mathrm{HGSnon}-\mathrm{dom} \%$ pred & $90 \pm 14$ & $77 \pm 12^{* *}$ \\
$\mathrm{HGSdom} \%$ pred & $87 \pm 24$ & $77 \pm 19^{*}$ \\
Daily physical activity au & $16.3 \pm 9.8$ & $13.1 \pm 6.2^{*}$ \\
\hline
\end{tabular}

Data are presented as mean \pm SD unless otherwise indicated. COPD: chronic obstructive pulmonary disease; BMI: body mass index; FEV1: forced expiratory volume in one second; FVC: forced vital capacity; TLC: total lung capacity; $D \mathrm{~L}, \mathrm{CO}$ : $\mathrm{CO}$ diffusing capacity of the lung; $P a, O_{2}$ : arterial $\mathrm{O}_{2}$ tension; $P \mathrm{a}, \mathrm{CO}_{2}: \mathrm{CO}_{2}$ arterial tension; $P \mathrm{I}$,max: maximal inspiratory pressure; HGSnon: nondominant hand grip strength; HGSdom: dominant hand grip strength; au: arbitrary units as in VOORRIPS et al. [20]; ND: not determined. *: $\mathrm{p}<0.05$; **: $\mathrm{p}<0.01$.

significant statistical difference in PA scores was observed between COPD patients and controls (table 1).

\section{Strength of the quadriceps muscle}

The values of QMVC showed a wide range in the two study groups (table 2). The control group showed a QMVC of 23$65 \mathrm{~kg}$. The COPD patients showed quadriceps weakness as

Table 2.-Strength and endurance of the dominant quadriceps

\begin{tabular}{lcc}
\hline & $\begin{array}{c}\text { Healthy elderly } \\
\text { controls }\end{array}$ & $\begin{array}{c}\text { COPD } \\
\text { patients }\end{array}$ \\
\hline QMVC kg & $49 \pm 13$ & $22 \pm 10^{* *}$ \\
QMVC \% pred & $110 \pm 29$ & $63 \pm 27^{* *}$ \\
QTlim min & $30 \pm 0$ & $6.9 \pm 5.3^{* *}$ \\
QTlim-final $f$ EMG \% initial & $99 \pm 12$ & $87 \pm 13^{* *}$ \\
QTlim- $V^{\prime} \mathrm{O}_{2}$ max $\mathrm{mL} \cdot \mathrm{kg}^{-1} \cdot \mathrm{min}^{-1}$ & $9.6 \pm 3.6$ & $8.5 \pm 2.2$ \\
QTlim- $\Delta V^{\prime} \mathrm{O}_{2}$ rest versus end $^{-1}$ & $242 \pm 197$ & $164 \pm 123$ \\
QTlim-HRmax beat $\cdot \mathrm{min}^{-1}$ & $118 \pm 22$ & $96 \pm 16^{* *}$ \\
QTlim-HRmax \% pred & $59 \pm 11$ & $63 \pm 14$ \\
QTlim- $\Delta$ HR rest versus end & $43 \pm 26$ & $22 \pm 23$ \\
QTlim- $V^{\prime}$ Emax L·min & -1 & $31 \pm 12$ \\
QTlim- $V^{\prime}$ Emax \% pred & $18 \pm 9$ & $55 \pm 6^{* *}$ \\
QTlim-dyspnoea end VAS & $1 \pm 1$ & $5 \pm 3 * *$ \\
QTlim-thigh discomfort VAS & $6 \pm 2$ & $10 \pm 0^{* *}$ \\
Thigh length cm & $39.9 \pm 1.0$ & $40.9 \pm 2.8$ \\
Thigh perimeter cm & $49.1 \pm 4.1$ & $44.6 \pm 6.4$ \\
\hline
\end{tabular}

Data are presented as mean $\pm \mathrm{SD}$. Both heart rate (HR) and minute ventilation $\left(V^{\prime} \mathrm{E}\right)$ values appear referenced to values from JONES et al. [33] for incremental cycle ergometry. COPD: chronic obstructive pulmonary disease; QMVC: quadriceps maximal strength during maximum voluntary contraction; QTlim: quadriceps endurance using a normalised exercise with a load equivalent to $10 \%$ of the QMVC and a contraction frequency of $12 \mathrm{~min}^{-1}$; $f$ EMG: median frequency of the electromyographic spectra; $V^{\prime} \mathrm{O}_{2}: \mathrm{CO}_{2}$ production; HRmax: maximum HR; $V^{\prime}$ Emax: maximum minute ventilation; VAS: visual analogic scale. $* *: \mathrm{p}<0.01$. 


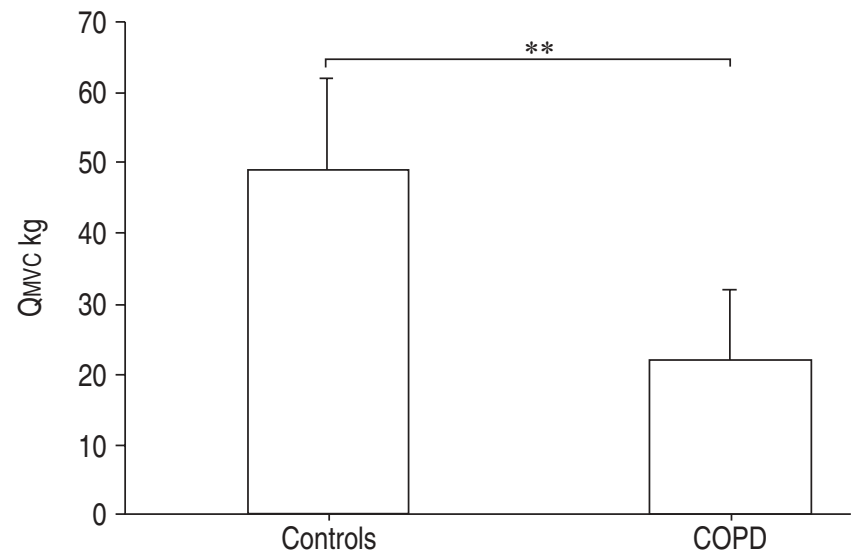

Fig. 1.- Mean \pm SD values of the quadriceps muscle strength as assessed by maximal voluntary contraction (QMVC). COPD: chronic obstructive pulmonary disease. ${ }^{* *}: \mathrm{p}<0.01$.

shown by a significant decrease of QMVC value ( $43 \%$ of control values, $\mathrm{p}<0.01$ ) (fig. 1 ).

\section{Endurance of the quadriceps muscle}

All healthy controls reached the 30-min time limit when performing the QTlim test (fig. 2a). In contrast, all COPD patients showed an early onset of task failure as represented by $\sim 77 \%$ lower QTlim when compared with control volunteers. This finding was evident even in those patients showing only mild or moderate airflow obstruction and relatively normal PA. Impairment in the QTlim was irrespective of daily PA scores (fig. 2b). Task failure was associated with limiting symptoms (discomfort) of the exercised thigh (fig. 3a), decrease of $f$ EMG (fig. $3 \mathrm{~b}$ ), and a transitory decrease in the quadriceps strength equivalent to $\sim 25 \%$ of initial QMVC after task failure (fig. 3c). This decrease of strength was partially recovered after $10 \mathrm{~min}$ of rest. Ventilatory and cardiac limitations were not observed during the endurance run as assessed by peak minute ventilation and heart rate values (table 2).

\section{Correlations}

Overlap between patients with COPD and the age-matched controls was found in strength measurements but not in endurance measurements. QMVC did not correlate with pulmonary function (fig. 4a), nutritional parameters or daily PA. A weak correlation was found between the degree of airflow obstruction $(\% \mathrm{FEV} 1)$ and $\mathrm{QT} \lim \left(\mathrm{R}^{2}=0.1532, \mathrm{p}<0.05\right)$ (fig. 4b). No correlation occurred between QMVC and QTlim (fig. 5). No association was found between QTlim and thigh perimeter or nutritional variables.

\section{Discussion}

The main finding of the present study was that skeletal muscle endurance was reduced in community-based noncomorbid COPD patients, even in those lacking sedentarism. Remarkably, the decrease in quadriceps endurance was present not only in patients with severe but also in those with mild or moderate airflow obstruction. Although impaired quadriceps endurance is weakly related to FEV1, it does not show any correlation with nutritional status, static lung
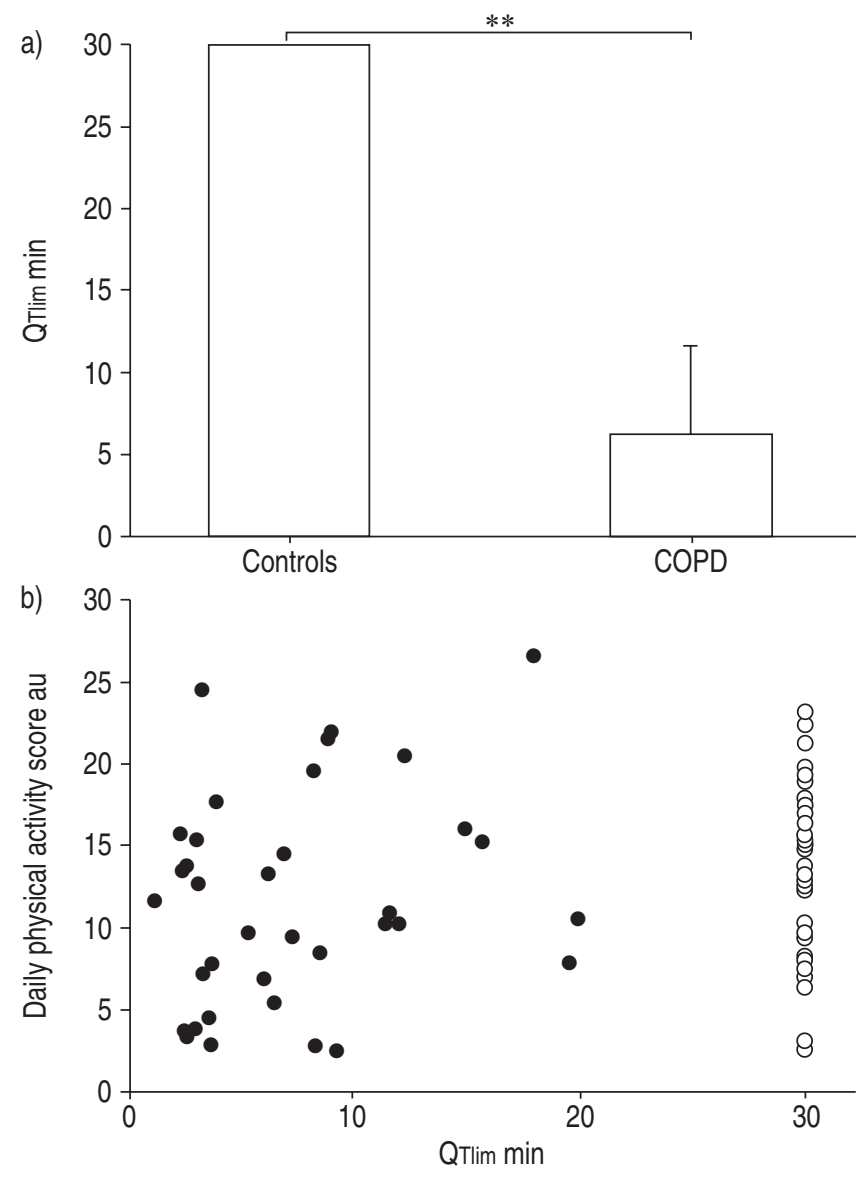

Fig. 2. - The quadriceps endurance test (QTlim) results of control and chronic obstructive pulmonary disease (COPD) subjects (mean \pm SD values (a)), and relationship with the daily physical activity score (b). $\bullet$ : COPD subjects; $\bigcirc$ : control subjects. au: arbitrary units. **: $\mathrm{p}<0.01$.

volumes, arterial blood gases or PA scores. The study confirms reduction in quadriceps strength in the COPD patients. However, muscle endurance is irrespective of muscle strength and is related to an increased susceptibility to peripheral muscle fatigue as task failure was associated with a decrease in $f$ EMG and transitory loss of strength in the exercised thigh.

Dysfunction of peripheral muscles has been described in association with other chronic diseases, such as heart and renal failure, AIDS and neoplasms. This suggests the existence of a common scenario in all these diseases. The most recent theories integrate the knowledge derived from diverse studies and propose that muscle dysfunction is the result of a group of factors with systemic effects (sedentarism [16], inflammatory cytokines [34] and growth factors [35]) interacting with a group of local factors (progressive inactivity, imbalance in the redox system [36] and acidosis [37, 38]).

Various investigators proposed that sedentarism may be one of the leading causes of impaired quadriceps function in COPD patients $[16,40,41]$. In the present study, all COPD patients showed increased susceptibility to fatigue, whereas all healthy controls showed tolerance to $30 \mathrm{~min}$ of exertion. All patients showed a combination of limiting symptoms, decreased $f$ EMG and transitory loss of strength in the exercised thigh at the end-point of the endurance test. This implies that the presence of the disease is associated with impaired skeletal muscle endurance.

The novel aspects of the present study can be summarised 

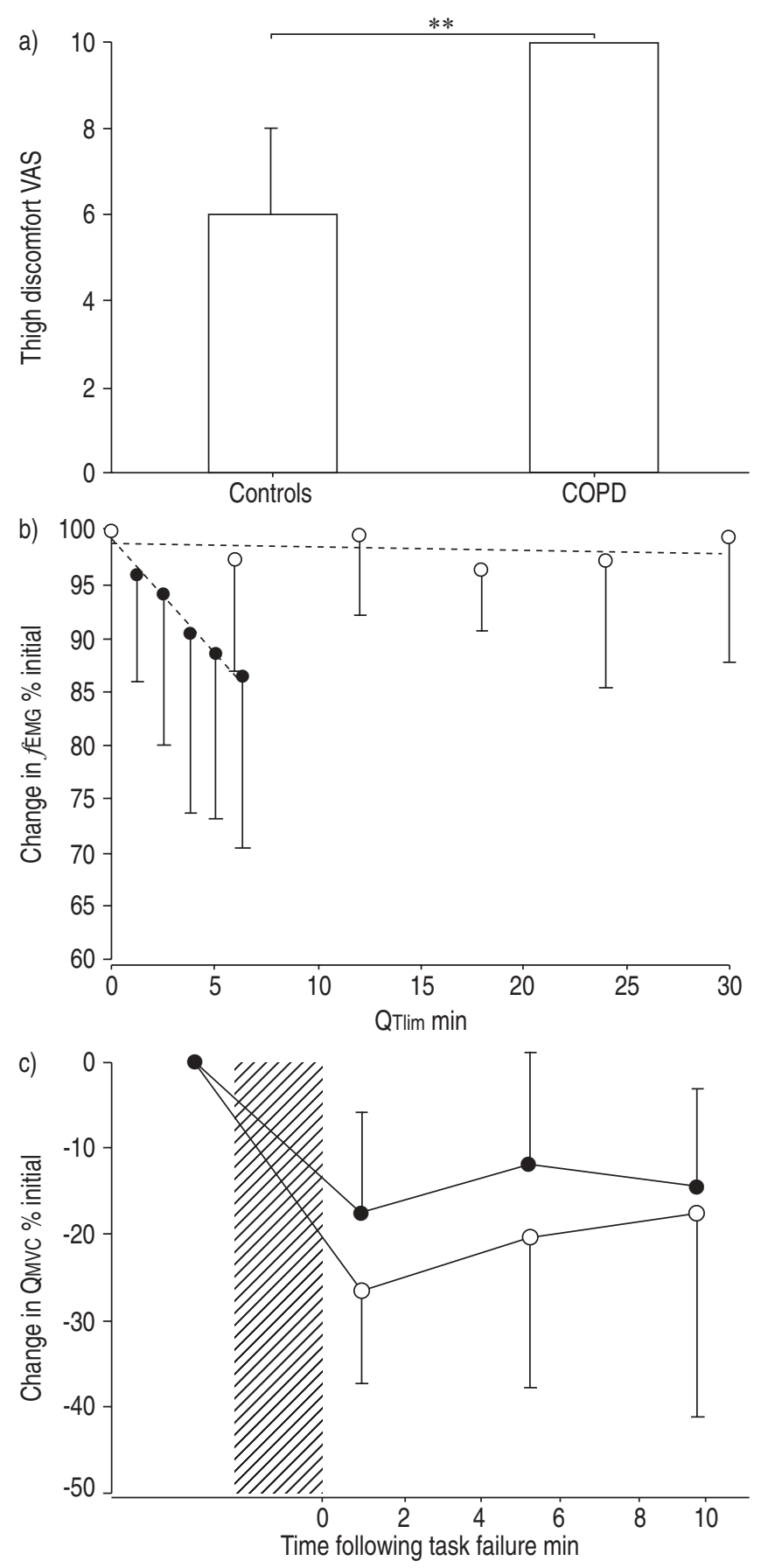

Fig. 3.-Results from endurance test of the quadriceps for a) values of perceived discomfort on the exercised leg at the end of the endurance exercise (mean $\pm \mathrm{SD}$ ), b) change in the median frequency of the recorded electromyography ( $f \mathrm{EMG}$ ) of the exercised quadriceps during the quadriceps endurance test $(Q$ Tlim) and c) mechanical evidence of muscle fatigue as assessed by change in maximum voluntary contractions (QMVC). VAS: visual analogic scale. $\mathbb{Z}$ : quadriceps endurance test. $\bullet$ : chronic obstructive pulmonary disease subjects; $\bigcirc$ : control subjects. ${ }^{* *}: \mathrm{p}<0.01$.

as four main concepts. The first concept is that impaired QTlim was found in patients with mild or moderate COPD. This finding implies that impaired skeletal muscle endurance develops even in early stages of the disease. The second point deals with the absence of difference in QTlim when the patients were stratified according to PA scores. The authors'
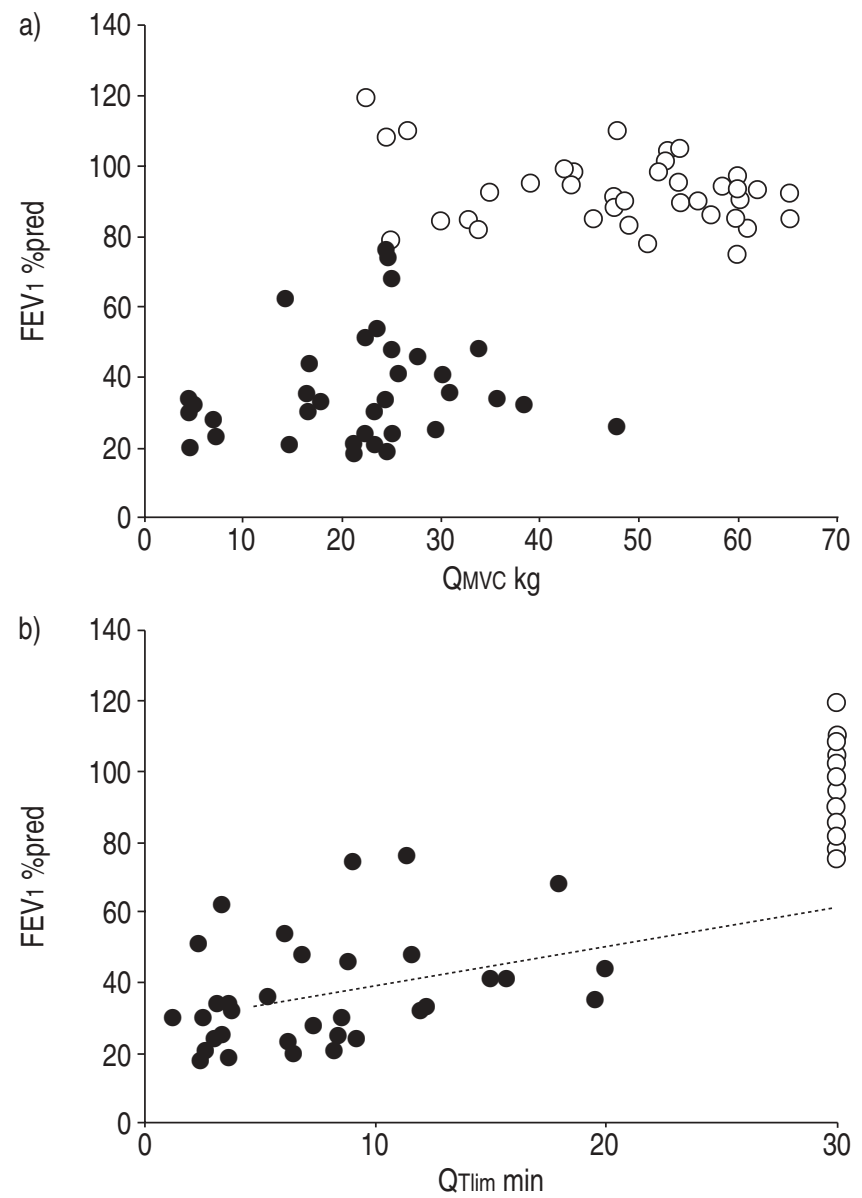

Fig. 4.-Relationship between the degree of airflow obstruction and function of the quadriceps muscle as assessed by a) maximal voluntary contraction (QMvC) and b) the quadriceps endurance test (QTlim). FEV1: forced expiratory volume in one second. $\bullet$ : chronic obstructive pulmonary disease subjects; $\bigcirc$ : control subjects. $\mathrm{p}<0.05$ COPD patient only.

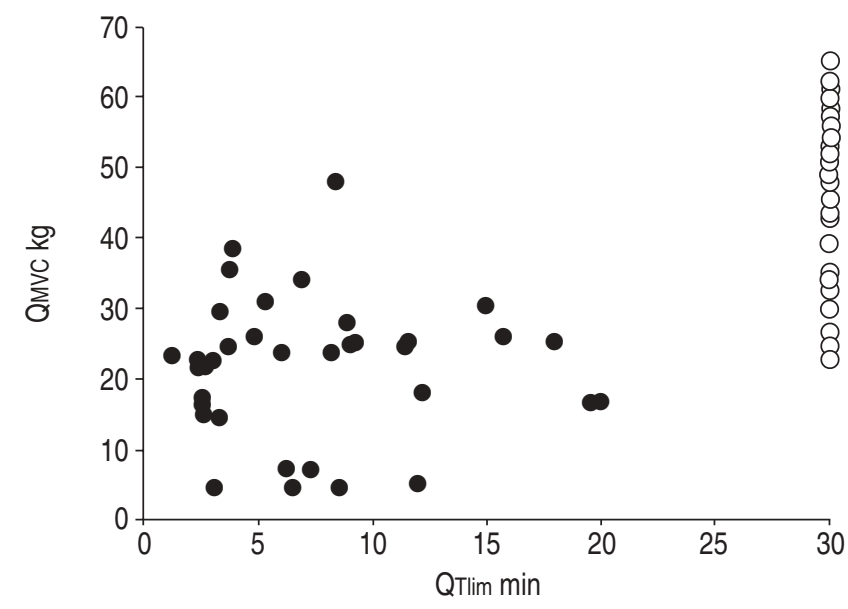

Fig. 5.-Scatter plot showing absence of linearity between the function of the quadriceps muscle as assessed by maximal voluntary contraction (QMVC) and the quadriceps endurance test (QTlim). chronic obstructive pulmonary disease subjects; $\bigcirc$ : control subjects.

novel contribution is that significant impairment in skeletal muscle endurance can be found even in patients without severe sedentarism, suggesting that other factors should be 
considered to explain the impairment of strength and endurance in the quadriceps from COPD. This result, which contrasts with those from SERRES et al. [16], is surprising since the same muscle group and the same questionnaire were used in the analyses. Their patients disclosed a severe decrease in their PA (lower PA scores), half of those obtained in the present study. The present authors hypothesise that cultural variables may not account for such a difference, since the impairment in pulmonary function is similar in these two studies and the ethnic background appears to be similar between Montpellier and Barcelona [42]. Daily activity levels as assessed by questionnaires allow the investigators to conduct retrospective estimations of activities. Other authors have also used the same questionnaire and consider it a reliable and valid method for classifying the activity levels of aged subjects [20, 21]. However, questionnaires have several limitations as the rate at which work is performed is seldom taken into account. This is relevant because as patients become more impaired, they reduce the intensity and prolong the duration of the work, an effective strategy in reducing symptom intensity. Although prospective and limited to a short period of time, complimentary ways of assessment could include pedometers or movement counters. The third concept relates to the magnitude of the load imposed for the quadriceps exercise. Some authors have suggested that healthy skeletal muscles can tolerate an exercise indefinitely if the TT index is $<0.15$ [43]. To the authors' knowledge, this is the first study showing an early onset of task failure in COPD patients exercising only one leg against a load equivalent to only $10 \%$ QMVC and a TTQ index consistently $<0.04$ (in spite of the absence of cardiac or ventilatory limitations during this specific exercise). The current authors modified the protocol described by SERRES et al. [16], which evaluated a load equivalent to $20 \%$ QMVC. In their study, most patients were able to perform just a few contractions (12 movements per min) for up to $3 \mathrm{~min}$ (i.e. $\sim 30$ muscle contractions). In the current authors' opinion these data are not optimal in order to analyse muscle endurance as a continuous variable in linear regression correlations. Finally, the fourth point deals with symptoms and spectral changes in the EMG. The analysis of peak cardioventilatory response shows that susceptibility to quadriceps exhaustion in COPD is present even in the absence of ventilatory or cardiac limitations. The present authors attempted to determine that peripheral factors are important by measuring EMG median frequency and QMVC after the endurance run. All COPD patients disclosed thigh discomfort as the limiting symptom related to task failure, which was associated with a significant reduction of $f \mathrm{EMG}$ and QMVC. The latter was partially recovered following $10 \mathrm{~min}$ of rest. These data support the theory that such exhaustion is not justified simply by perceptual factors during the exercise, but also by contractile fatigue.

Submaximal power sustained over time is always associated with changes in the relationship between motor command and power in single motor units. At a subjective level, the effort required to sustain power increases systematically with power and time. It is possible that the fall in QMVC following the endurance run could also be related in part to a decrease in activation, and that this change was larger in the patients with COPD because leg symptoms were prominent in this group. However, the current authors acknowledge that the relationship between changes in EMG median frequency and contractile fatigue is controversial. No definite answers can be drawn from the present study. Measurement of voluntary QMVC has some limitations due to the potential differences in the degree of activation during the manoeuvre. Additional research using twitch stimulation may give further insight into potential contractile fatigue.

Several studies have shown a significant decrease in the size of fibres, capillary density, proportion of fibres with oxidative metabolism and aerobic enzyme activity within the vastus lateralis muscle from patients with COPD [44-46]. Functionally, these structural changes could be related to both weakness and susceptibility to fatigue of the quadriceps muscle, during whole body or specific aerobic exercise [5, 47]. The current authors found that weakness and early onset of fatigue was also present even in patients with similar thigh perimeter to controls. However, thigh perimeter could not have been sensitive enough to detect changes in the amount of muscle, concomitant with reciprocal changes in fat distribution [3, 39, 40].

A potential limitation is that QTlim data were analysed in absolute values and some reference values are available for quadriceps strength but not for endurance. In addition, quadriceps biopsies were not taken due to the fact that this investigation was not designed as a structural study. Some alternative approaches could be the metabolic study of the quadriceps muscle using catheterisation of femoral artery and vein, infrared spectrometry to assess local metabolism, and imaging techniques (i.e. nuclear magnetic resonance or computerised tomography) to assess the mass of the quadriceps muscle. Anthropometry and serum protein analysis were unable to detect significant differences in the nutritional status of the COPD patients. In addition, the body weight, $\mathrm{BMI}$ and thigh perimeter were found to be preserved in the COPD group. These findings suggest that quadriceps strength and endurance can be significantly impaired even in the absence of notable weight loss.

\section{Potential clinical implications}

The present study shows that impaired muscle function is present even in COPD patients showing normal weight, lacking respiratory failure and lacking comorbidity. In consonance with CLARK et al. [48] the present results suggest that impaired quadriceps endurance should not be considered solely a feature of advanced COPD, drugs, starvation or comorbidity. It is known that the decline in power (or strength) with the duration of activity vary with endurance from subject to subject because the physiological factors limiting power over time change substantially. The present study shows that there are obvious differences in the COPD group. In addition, the present data show that dysfunction of the quadriceps muscle in patients with COPD can be underestimated by measures of strength only. In fact, quadriceps endurance cannot be predicted based on strength measurements. All subjects performed the specific quadriceps exercise against the same proportion of maximal voluntary contraction. Comparisons of COPD and controls at a constant percentage of their strength probably underscore the severity of the impairment.

Exercise is an integrated activity that has to be analysed from the point of view of system analysis. Although limiting factors can be studied in a single sphere (e.g. peripheral muscles), they must be interpreted in the light of the total effects on the system in order to understand the phenomena at a broader level. From a clinical point of view, these data confirm that specific quadriceps endurance tests offer additional information, with potential clinical impact, and should be considered complimentary to the quadriceps strength and general exercise evaluations. The current authors believe that this and some previous studies emphasise the potential clinical relevance of assessing quadriceps endurance in COPD patients using a relatively cheap, user-friendly technology that is readily available in most physiopathology and rehabilitation laboratories. Local (specific) limb exercise may contribute 
to obviate potential bias related to ventilatory and cardiac limitation, which are intrinsic to whole-body exercise protocols $[16,49]$.

\section{Conclusions}

This study illustrates that normal weight and clinically stable chronic obstructive pulmonary disease patients show a marked impairment in the endurance of the quadriceps muscle. The study shows that this impairment, irrespective of physical activity, is present even in patients with mild-tomoderate airflow obstruction. Endurance of the muscle cannot be predicted from the severity of the disease or the reduction in quadriceps strength. The exhaustion during local exercise is related to early onset of muscle fatigue. These findings suggest that not only deconditioning, but also other factors related to chronic obstructive pulmonary disease are determinants of muscle endurance.

\footnotetext{
Acknowledgements. The authors gratefully acknowledge L. Lindström for his useful suggestions, and A. Roig and N. Soler for their technical support.
}

\section{References}

1. Dugan SA, Frontera WR. Muscle fatigue and muscle injury. Phys Med Rehabil Clin N Am 2000; 11: 385-403.

2. Coyle EF. Physiological determinants of endurance exercise performance. J Sci Med Sport 1999; 2: 181-189.

3. Gosselink R, Troosters T, Decramer M. Peripheral muscle weakness contributes to exercise limitation in COPD. $\mathrm{Am}$ J Respir Crit Care Med 1996; 153: 976-980.

4. Bauerle O, Chrusch CA, Younes M. Mechanisms by which COPD affects exercise tolerance. Am J Respir Crit Care Med 1998; 157: 57-68

5. Rabinovich R, Vilaro J, Roca J. The role of peripheral muscles on exercise tolerance in patients with COPD. Arch Bronconeumol 2001; 37: 135-141.

6. Garrod R, Bestall JC, Paul EA, Wedzicha JA, Jones PW. Development and validation of a standardized measure of activity of daily living in patients with severe COPD: the London Chest Activity of Daily Living scale (LCADL). Respir Med 2000; 94: 589-596.

7. Maltais F, Jobin J, Sullivan MJ, et al. Metabolic and hemodynamic responses of lower limb during exercise in patients with COPD. J Appl Physiol 1998; 84: 1573-1580.

8. Ferrer M, Alonso A, Morera $\mathrm{J}$, et al. Chronic obstructive pulmonary disease stage and health-related quality of life. The Quality of Life of Chronic Obstructive Pulmonary Disease Study Group. Ann Intern Med 1997; 127: 1072-1979.

9. Yohannes AM, Roomi J, Waters K, Connolly MJ. Quality of life in elderly patients with COPD: measurement and predictive factors. Respir Med 1998; 92: 1231-1236.

10. Decramer M, Gosselink R, Troosters T, Verschueren M, Evers G. Muscle weakness is related to utilization of health care resources in COPD patients. Eur Respir J 1997; 10: 417423.

11. Engelen MP, Schols AM, Does JD, Wouters EF. Skeletal muscle weakness is associated with wasting of extremity fatfree mass but not with airflow obstruction in patients with chronic obstructive pulmonary disease. Am J Clin Nutr 2000; 71: 733-738.

12. Lands LC, Smountas AA, Mesiano G, et al. Maximal exercise capacity and peripheral skeletal muscle function following lung transplantation. $J$ Heart Lung Transplant 1999; 18: 113-120.
13. Trulock EP. Lung transplantation for COPD. Chest 1998; 113: 269-276.

14. Starkey DB, Pollock ML, Ishida Y, et al. Effect of resistance training volume on strength and muscle thickness. Med Sci Sports Exerc 1996; 28: 1311-1320.

15. Hamilton AL, Killian KJ, Summers E, Jones NL. Muscle strength, symptom intensity, and exercise capacity in patients with cardiorespiratory disorders. Am J Respir Crit Care Med 1995; 152: 2021-2031.

16. Serres I, Gautier V, Varray A, Prefaut C. Impaired skeletal muscle endurance related to physical inactivity and altered lung function in COPD patients. Chest 1998; 113: 900-905.

17. Siafakas NM, Vermeire $\mathrm{P}$, Pride NB, et al. Optimal assessment and management of chronic obstructive pulmonary disease (COPD). Eur Respir J 1995; 8: 1398-1420.

18. Barberà JA, Peces-Barba G, Agustí AGN, et al. Clinical guidelines for the diagnosis and treatment of chronic obstructive pulmonary disease. Arch Bronconeumol 2001; 37: 297-316.

19. Baecke JAH, Burema J, Frijters JER. A short questionnaire for the measurement of habitual physical activity in epidemiological studies. Am J Clin Nutr 1982; 36: 936-942.

20. Voorrips LE, Ravelli ACJ, Dongelmans PCA, Deurenberg $\mathrm{P}$, Van Staveren WA. A physical activity questionnaire for the elderly. Med Sci Sports Exerc 1991; 23: 974-979.

21. Couillard A, Koechlin C, Cristol JP, Varray A, Prefaut C. Evidence of local exercise-induced systemic oxidative stress in chronic obstructive pulmonary disease patients. Eur Respir J 2002; 20: 1123-1129.

22. Bink B, Bonjer H, Van Der Sluys H. Assessment of the energy expenditure by indirect time and motion study. In: Edang K, Lange Andersen K, eds. Physical activity in health and disease. Proceedings of the Bertostölen Symposium, Oslo, Oslo University, 1966; pp. 207-214.

23. Roca J, Sanchis J, Agusti-Vidal A, et al. Spirometric reference values from a Mediterranean population. Bull Eur Physiopathol Respir 1986; 22: 217-224.

24. Roca J, Burgos F, Barbera JA, et al. Prediction equations for plethysmographic lung volumes. Respir Med 1998; 92: 454460.

25. Roca J, Rodriguez-Roisin R, Cobo E, Burgos F, Perez J, Clausen JL. Single-breath carbon monoxide diffusing capacity prediction equations from a Mediterranean population. Am Rev Respir Dis 1990; 141: 1026-1032.

26. Morales P, Sanchis J, Lamb PJ, Diez JL. Maximum static respiratory pressures in adults. The reference values for a Mediterranean Caucasian population. Arch Bronconeumol 1997; 33: 213-219.

27. Mathiowetz V, Dove M, Kashman N, Rogers S. Grip and pinch strength: normative data for adults. Arch Phys Med Rehabil 1985; 66: 69-72.

28. Peolsson A, Hedlund R, Öberg B. Intra- and inter-tester reliability and reference values for hand strength. J Rehab Med 2001; 33: 36-41.

29. Decramer M, Lacquet LM, Fagard R, Rogiers P. Corticosteroids contribute to muscle weakness in chronic airflow obstruction. Am J Respir Crit Care Med 1994; 150: 11-16.

30. Mahler DA, Mejía-Alfaro R, Ward J, Baird JC. Continuous measurement of breathlessness during exercise: validity, reliability, and responsiveness. J Appl Physiol 2001; 90: 2188-2196.

31. Lindström L, Kadefors R, Petersén I. An electromyographic index for localized muscle fatigue. J Appl Physiol Respirat Environ Exercise Physiol 1977; 43: 750-754.

32. Van Dijk JG. Influence of different types of surface electrodes on amplitude, area and duration of the compound muscle action potential. Clin Neurophysiol 2000; 111: 17061708.

33. Jones NL, Summers E, Killian KJ. Influence of age and stature during incremental cycle ergometry in men and women. Am Rev Respir Dis 1989; 140: 1373-1380.

34. Eid AA, Ionescu AA, Nixon LS, et al. Inflammatory 
response and body composition in chronic obstructive pulmonary disease. Am J Respir Crit Care Med 2001; 164: 1414-1418.

35. Hodge S, Hodge G, Holmes M, Flower R, Scicchitano R. Interleukin-4 and tumour necrosis factor-alpha inhibit transforming growth factor-beta production in a human bronchial epithelial cell line: possible relevance to inflammatory mechanisms in chronic obstructive pulmonary disease. Respirology 2001; 6: 205-211.

36. Rabinovich RA, Ardite E, Troosters T, et al. Reduced muscle redox capacity after endurance training in patients with chronic obstructive pulmonary disease. Am J Respir Crit Care Med 2001; 164: 1114-1118.

37. Maltais F, Simard AA, Simard C, Jobin J, Desgagnes P, LeBlanc P. Oxidative capacity of the skeletal muscle and lactic acid kinetics during exercise in normal subjects and in patients with COPD. Am J Respir Crit Care Med 1996; 153: 288-293.

38. Gea J, Orozco-Levi M, Barreiro E, Ferrer A, Broquetas J. Structural and functional changes in the skeletal muscles of COPD patients: the "compartments" theory. Monaldi Arch Chest Dis 2001; 56: 214-224.

39. Debigare $\mathrm{R}$, Cote $\mathrm{CH}$, Maltais F. Peripheral muscle wasting in chronic obstructive pulmonary disease. Clinical relevance and mechanisms. Am J Respir Crit Care Med 2001; 164: 1712-1717.

40. Mador MJ, Kufel TJ, Pineda L. Quadriceps fatigue after cycle exercise in patients with chronic obstructive pulmonary disease. Am J Respir Crit Care Med 2000; 161: 447-453.
41. Bernard S, LeBlanc P, Whittom F, et al. Peripheral muscle weakness in patients with chronic obstructive pulmonary disease. Am J Respir Crit Care Med 1998; 158: 629-634.

42. Coronell C, Orozco-Levi M, Gea J. COPD and body weight in a Mediterranean population. Clin Nutr 2002; 21: 437-438.

43. Monod H, Lemaire C, Metral S. Tension-length diagram and electromyogram of carpal extensor muscles. Ann Chir 1976; 30: 931-933.

44. Jobin J, Maltais F, Doyon JF, et al. Chronic obstructive pulmonary disease: capillarity and fiber-type characteristics of skeletal muscle. J Cardiopulm Rehabil 1998; 18: 432-437.

45. Maltais F, LeBlanc $\mathrm{P}$, Whittom $\mathrm{F}$, et al. Oxidative enzyme activities of the vastus lateralis muscle and the functional status in patients with COPD. Thorax 2000; 55: 848-853.

46. Maltais F, Sullivan MJ, LeBlanc P, et al. Altered expression of myosin heavy chain in the vastus lateralis muscle in patients with COPD. Eur Respir J 1999; 13: 850-854.

47. Booth FW, Thomason DB. Molecular and cellular adaptation of muscle in response to exercise: perspectives of various models. Physiol Rev 1991; 71: 541-585.

48. Clark CJ, Cochrane LM, Mackay E, Paton B. Skeletal muscle strength and endurance in patients with mild COPD and the effects of weight training. Eur Respir J 2000; 15: 92 97.

49. Montes de Oca M, Ortega Balza M, Lezama J, Lopez JM. Chronic obstructive pulmonary disease: evaluation of exercise tolerance using three different exercise tests. Arch Bronconeumol 2001; 37: 69-74. 